\title{
Characterization and stability of lactobacilli and yeast microbiota in kefir grains
}

\author{
T. Vardjan, ${ }^{* 1}$ P. Mohar Lorbeg, $†$ I. Rogelj, $†$ and A. Čanžek Majhenič† \\ ${ }^{*}$ Kele \& Kele d.o.o., Laze 22a, 1370 Logatec, Slovenia \\ †Biotechnical Faculty, Department of Animal Science, Institute of Dairy Science and Probiotics, University of Ljubljana, Groblje 3, \\ 1230 Domžale, Slovenia
}

\begin{abstract}
Characterization and stability of lactobacilli and yeasts from kefir grains using culture-dependent and culture-independent methods were investigated in this study. Culture-dependent analysis, followed by sequencing of $16 \mathrm{~S}$ ribosomal DNA for bacteria and $26 \mathrm{~S}$ rRNA gene for yeasts, revealed 3 different species of lactobacilli and yeasts, respectively. The most frequently isolated bacterial species were Lactobacillus kefiranofaciens ssp. kefirgranum, Lb. parakefiri, and Lb. kefiri, whereas yeasts belonged to Kluyveromyces marxianus, Kazachstania exigua, and Rhodosporidium kratochvilovae. This study is the first to report on the presence of $R$. kratochvilovae in kefir grains. On the other hand, PCR-denaturing gradient gel electrophoresis in the culture-independent method showed that the dominant microorganisms were $L b$. kefiranofaciens ssp. kefirgranum, Kl. marxianus and Ka. exigua, but did not reveal bands corresponding to Lb. parakefiri, $L b$. kefiri, or R. kratochvilovae. Our results support the necessity of combining more techniques for detailed and reliable study of microbial communities in kefir grains. Another interesting finding confirmed that the detected dominant microbiota of kefir grains is very stable and did not change over experimental time. This finding is important to ensure consistent product quality.

Key words: kefir grain, lactobacilli, yeast
\end{abstract}

\section{INTRODUCTION}

Kefir is a traditional fermented milk originating from the Caucasus mountains. It is a self-carbonated dairy product with a slightly acidic taste, yeasty flavor, creamy consistency, and low percentage of alcohol (Marshall, 1993; Farnworth, 2005; Garrote et al., 2010). Many health benefits have been attributed to kefir, including the enhancement of the immune system

Received June 12, 2012.

Accepted January 13, 2013.

${ }^{1}$ Corresponding author: tinkara.vardjan@bf.uni-lj.si and improvement of digestive health, as well as antimicrobial, antitumoral, antiviral, antimutagenic, and antioxidant activity (Otes and Cagindi, 2003; Santos et al., 2003; Liu et al., 2005; Rodrigues et al., 2005; de Moreno de LeBlanc et al., 2007). Kefir is produced by the fermentation of milk with kefir grains that represent complex and dynamic microbial symbiosis of lactic acid bacteria (lactobacilli, lactococci, leuconostoc, streptococci), yeasts, and acetic acid bacteria. The most common lactobacilli isolated from kefir grains are Lactobacillus kefiri, Lactobacillus kefiranofaciens, Lactobacillus kefirgranum, Lactobacillus parakefiri, Lactobacillus delbrueckii, Lactobacillus acidophilus, Lactobacillus brevis, Lactobacillus helveticus, Lactobacillus casei, Lactobacillus paracasei, Lactobacillus fermentum, Lactobacillus plantarum, and Lactobacillus gasseri (Angulo et al., 1993; Marshall, 1993; Takizawa et al., 1998; Garrote et al., 2001; Simova et al., 2002; Zhou et al., 2009; Da Cruz Pedrozo Miguel et al., 2010). Kefir-specific yeasts play a key role in the formation of flavor and aroma. They are represented by the species Kluyveromyces marxianus, Kluyveromyces lactis, Saccharomyces cerevisiae, Torulaspora delbrueckii, Candida kefir, Pichia fermentans, Kazachstania unispora, and Kazachstania exigua (Angulo et al., 1993; Marshall, 1993; Garrote et al., 2001; Simova et al., 2002; Zhou et al., 2009). Witthuhn et al. (2004) indicated that kefir grain microbiota strongly depends on the origin of the grains, the local conditions of culture, and the storage and manipulation processes. The growth and survival of individual strains in kefir grains are dependent on the presence of others; because of this, kefir grains are a good example of symbiosis. When the various microorganisms are separated as pure cultures, they do not grow in milk or have decreased biochemical activity. The growth of several bacteria isolated from kefir grains was improved when yeast extract was added to growth medium, indicating that the yeasts found in kefir grains were essential to maintain the integrity and viability of the microbiota population. Vitamins, $\mathrm{AA}$, and other essential growth factors for bacteria are produced by yeasts, whereas bacterial metabolic end 
products are used as energy sources by yeasts (Viljoen, 2001; Farnworth and Mainville, 2003).

Kefir grains serve as basic inoculums in traditional kefir production, but their complex microbiological association makes it difficult to obtain defined and constant kefir starter culture appropriate for industrial kefir production of conventional properties (Robinson et al., 2002). Therefore, in recent years, attention has been focused on producing kefir from a mixture of pure and defined cultures. This enables better control over the microorganisms involved and greater ease of production, but the properties of the final product significantly differ from kefir fermented with kefir grains (Marshall, 1993; Özer and Özer, 1999; Beshkova et al., 2002). Koroleva (1988) cautioned that any attempt to replace kefir grains by a mixture of pure microorganisms would not be effective because the unique composition of kefir grains that has evolved over time cannot be replicated or replaced. Any symbiotic relationship between bacteria and yeasts that has developed over time is special; but for large-scale production of kefir with kefir grains, it is important that the microbiota of kefir grains is stable and does not change over time. Only in this way can we be sure of a product of the required and consistent quality that fulfils the criteria for traditional kefir.

Detecting and identifying the microbial composition of kefir grains and kefir with a rapid method is often important for quality control of this product. Moreover, complete information regarding microbiota composition can enlighten the mechanisms and interactions involved in the production of several bioactive materials, particularly those responsible for kefir grain formation. For many years, research on the microbiota in foods has only relied on conventional culture-dependent methods that employ isolation and cultivation of microorganisms before their morphological, biochemical, or genetic identification (Hugenholtz et al., 1998). Development of molecular technologies has enabled application of various differentiating methods for microbial identification based on genomic traits. Randomly amplified polymorphic DNA PCR (RAPD-PCR) was performed to characterize and type microorganisms isolated from kefir grains (Mainville et al., 2006). But this technique was based on culture-dependent steps, which are time-consuming, due to long cultivating periods and elaborate culture techniques. Furthermore, the results can often be influenced by the poor viability or low density of a given organism. For this reason, cultureindependent analyses have proven to be powerful tools in providing a more complete inventory of the microbial diversity in food samples (Dahllöf et al., 2000). Recently, PCR-denaturing gradient gel electrophoresis (PCR-DGGE) has been recognized as one of the most suitable and widely applied techniques to study complex microbial communities in food samples and other environments, such as kefir grains and kefir (Chen et al., 2008; Zhou et al., 2009; Da Cruz Pedrozo Miguel et al., 2010).

As previously mentioned, the composition of the kefir grains microbiota strongly depends on the origin of kefir grains as well as the local conditions of their manipulation. Therefore, the aim of this study was to evaluate the diversity and stability of lactobacilli and yeasts present in kefir grains from local dairy plant, using culture-dependent and culture-independent methods.

\section{MATERIALS AND METHODS}

\section{Kefir Grains}

Kefir grains were obtained from the local Mlekarna Krepko dairy plant (Kele \& Kele d.o.o., Logatec, Slovenia). In 1992, live kefir grains were brought from former Soviet Union countries by Dunja and Branislav Kelečević, founders and owners of the Mlekarna Krepko dairy plant. Since then, these same original kefir grains have been maintained and propagated for kefir production in the traditional way. To attain general information about composition and stability of lactobacilli and yeasts population, kefir grains were sampled 4 times, once per month. Between 2 consecutive samplings, kefir grains were transferred daily and propagated at $23^{\circ} \mathrm{C}$ in pasteurized milk according to dairy routine protocol. For analyses, kefir grains were aseptically prepared as follows: $10 \mathrm{~g}$ of kefir grains was suspended in $90 \mathrm{~g}$ of sterile trisodium citrate dihydrate $(20 \mathrm{~g} / \mathrm{L}, \mathrm{pH} 7.5$; Kemika, Zagreb, Croatia) and homogenized in BagMixer 400 (Interscience, St-Nom, France) for $12 \mathrm{~min}$. To determine composition and stability of lactobacilli and yeasts, homogenized samples of kefir grains were immediately subjected to culture-dependent and cultureindependent analyses.

\section{Bacterial and Yeasts Strains}

Type strains $L b$. kefiranofaciens ssp. kefiranofaciens LMG $19149^{\mathrm{T}}$ and Lb. kefiri LMG $9480^{\mathrm{T}}$ were purchased from Belgian Co-ordinated Collections of Microorganisms (Gent, Belgium), whereas Lb. kefiranofaciens ssp. kefirgranum DSM $10550^{\mathrm{T}}$ was purchased from Deutsche Sammlung von Mikroorganismen und Zellkulturen (Braunschweig, Germany). All lactobacilli were cultured in de Man, Rogosa, and Sharpe (MRS) broth (Merck, Darmstadt, Germany) under anaerobic conditions, using anaerobic jars and GENbox system (Bio-Merieux, Marcy-L'Etoile, France) at $30^{\circ} \mathrm{C}$ for $3 \mathrm{~d}$. 
The reference yeasts strains, S. cerevisiae CBS 1171, T. delbrueckii CBS 1146, and Ka. exigua CBS 379, were purchased from Fungal Biodiversity Centre (Utrecht, the Netherlands), whereas Ka. unispora NRRL Y-1556 was purchased from ARS Culture Collection (Peoria, IL) and Kl. marxianus IM444 was obtained from the collection of the University of Ljubljana, Biotechnical Faculty. All yeasts were cultured with Kluyveromyces differential medium (KDM; Valderrama et al. 1999) and incubated under aerobic conditions at $25^{\circ} \mathrm{C}$ for $5 \mathrm{~d}$. Type and reference strains were used for identification of lactobacilli and yeasts and isolated from kefir grains by culture-independent method.

\section{Isolation and Identification of Lactobacilli and Yeasts from Kefir Grains by Culture-Dependent Methods}

Decimal dilutions of homogenized samples of kefir grains were plated on MRS ( $\mathrm{pH} 5.2$ ) and KPL agar (Toba et al., 1986), and KDM agar for isolation of lactobacilli and yeasts, respectively. The KPL was composed of lactic acid whey $(1,000 \mathrm{~mL})$, white table wine $(70 \mathrm{~mL} / \mathrm{L})$, glucose $(10 \mathrm{~g} / \mathrm{L})$, galactose $(10 \mathrm{~g} / \mathrm{L})$, tween $80(1 \mathrm{~mL} / \mathrm{L})$, and agar $(15 \mathrm{~g} / \mathrm{L})$. Lactobacilli were incubated anaerobically, using anaerobic jars and GENbox system at $30^{\circ} \mathrm{C}$ for $3 \mathrm{~d}$, whereas yeasts were incubated aerobically for $5 \mathrm{~d}$ at $25^{\circ} \mathrm{C}$. After incubation, plates with a range of 30 to $300 \mathrm{cfu}$ were selected for further analysis. To determine the diversity of lactobacilli and yeasts from kefir grains, between 5 to 10 colonies were randomly picked from countable plates and transferred to MRS broth ( $\mathrm{pH}$ 5.2) and KDM broth, respectively; pure cultures were Gram stained.

After purification and microscopic examination, bacterial and yeast DNA from pure cultures was extracted using a Wizard Genomic DNA Purification Kit (Promega, Madison, WI). The pellet was first resuspended in $600 \mu \mathrm{L}$ of $50 \mathrm{mM}$ EDTA, containing $5 \mathrm{mg} / \mathrm{mL}$ of lysozyme, $25 \mathrm{U} / \mathrm{mL}$ of mutanolysin, and $12.5 \mu \mathrm{L} / \mathrm{mL}$ of lyticase (Sigma-Aldrich, Steinheim, Germany), and incubated at $37^{\circ} \mathrm{C}$ for $2 \mathrm{~h}$. Subsequent DNA purification steps were performed in accordance with the manufacturer's instructions. Strain typing of lactobacilli and yeasts was performed by RAPD-PCR using primers 1254 (5'-CCGCAGCCAA-3') and M13 (5'-GAGGGTGGCGGTTCT-3'), respectively. PCR programs used for 1254 and M13 were previously described by Torriani et al. (1999) and Andrighetto et al. (2000), respectively.

Species identification of isolated lactobacilli was confirmed by partial sequencing of the $16 \mathrm{~S}$ ribosomal (r) DNA. An approximately $660 \mathrm{bp}$ fragment of the $16 \mathrm{~S}$ rDNA was amplified using forward primer P1 (5'-GCGGCGTGCCTAATACATGC-3') and reverse primer P4
(5'-ATCTACGCATTTCACCGCTAC-3') (Klijn et al., 1991). For yeast identification, a $250 \mathrm{bp}$ fragment of the $26 \mathrm{~S}$ rRNA gene were amplified using primer pair NLc1 (5'-GCCATTTCAATAAGCGGCGGAAAA-3' and LS2 (5'-ATTCCCAAACAACTCGACTC-3'; Zhou et al., 2009). All sequencing analyses were performed by Microsynth (Balgach, Switzerland). Sequence homologies were analyzed by using BLAST database from GenBank (http://blast.ncbi.nlm.nih.gov/blast/).

\section{Screening of Lactobacilli and Yeasts from Kefir Grains by Culture-Independent Method - Denaturing Gradient Gel Electrophoresis (PCR-DGGE)}

One milliliter of homogenized samples of kefir grains was transferred into a plastic tube and the DNA was extracted using Maxwell 16 Cell DNA Purification Kit (Promega). Prior DNA extraction $1 \mathrm{~mL}$ of homogenate was first incubated for $2 \mathrm{~h}$ at $37^{\circ} \mathrm{C}$ in $600 \mu \mathrm{L}$ of Tris-EDTA buffer containing lysozyme $(5 \mathrm{mg} / \mathrm{mL})$, mutanolysin $(25 \mathrm{U} / \mathrm{mL})$, and lyticase $(12.5 \mu \mathrm{L} / \mathrm{mL})$. The suspension was transferred into the cartridge of the Maxwell 16 Instruments and further treated according to the manufacturer's instructions (Promega). The DNA was finally resuspended in $300 \mu \mathrm{L}$ of elution buffer containing 1.5 $\mu \mathrm{L}$ RNA-se $(4 \mathrm{mg} / \mathrm{mL})$. The bacterial DNA was amplified with universal primers HDA1-GC (5'-CGC CCG GGG CGC GCC CCG GGC GGG GCG GG GGC ACG GGG GGA CTC CTA CGG GAG GCA GCA GT-3'; GC clamp is underlined) and HDA2 (5'-GTA TTA CCG CGG CTG CTG GCAC-3'), spanning the $\mathrm{V} 2-\mathrm{V} 3$ region of the $16 \mathrm{~S}$ rDNA (Walter et al., 2000). Yeast DNA was amplified using primers NL1 (5'-CGC CCG CCG CGC GCG GCG GGC GGG GCG GGG GCC ATA TCA ATA AGC GGA GGA AAAG-3'; the GC clamp is underlined) and LS2 (5'-ATT CCC AAA CAA CTC GAC TC-3'), spanning the D1 region of the 26S rRNA gene (Cocolin et al., 2002).

Amplicons were separated using DGGE (D GENE system, Bio-Rad, Hercules, CA) with direct loading onto $8 \%$ (vol/vol) polyacrylamide gel with denaturating gradient 30 to $70 \%$ for primer pair HDA1-GC/HDA2, and 30 to $60 \%$ for primer pair NL1/LS2. The gradient was created using polyacrylamide (Sigma-Aldrich, St. Louis, MO) containing 0 to $100 \%$ of the denaturant [100\% corresponds to $7 \mathrm{M}$ urea (Sigma-Aldrich) and $40 \%$ (vol/vol) formamide (Sigma-Aldrich)]. Electrophoresis was performed at constant voltage of $75 \mathrm{~V}$ for 16 $\mathrm{h}$ at $60^{\circ} \mathrm{C}$. After electrophoresis, gels were stained in SYBR Safe (Invitrogen, Carlsbad, CA), photographed with UV transilluminator (Syngene, Cambridge, UK) and analyzed with BioNumerics software package (Applied Maths Inc., Austin, TX). 


\section{RESULTS AND DISCUSSION}

\section{Characterization of Lactobacilli and Yeasts in Kefir Grains Using Culture-Dependent Method}

The enumeration values obtained on MRS agar at first, second, third, and fourth sampling was 7.36, 7.38, 7.43 , and $7.40 \log \mathrm{cfu} / \mathrm{g}$, respectively. The number of colonies developed on KPL agar was 6.88, 6.91, 6.94, and $6.83 \mathrm{log} \mathrm{cfu} / \mathrm{g}$ at first, second, third, and fourth sampling, respectively. Similar lactobacilli count in kefir grains has been previously reported (Witthuhn et al., 2004). We found that the number of lactobacilli of kefir grains was remarkably stable and did not change over serial sampling. Analysis by MRS and KPL agar of 4 homogenized kefir grains samples revealed that 78 out of 88 total isolates belonged to genus Lactobacillus, and the remaining 10 isolates were cocci. Morphological characteristics of colonies distinguished these isolates into 3 groups; all 78 isolates were further subjected to RAPD-PCR fingerprinting to estimate lactobacilli diversity of kefir grains. The RAPD-PCR patterns of isolates within the same group were very similar. Between 6 and 9 isolates were randomly selected from each group, and a total of 23 isolates were subjected to $16 \mathrm{~S}$ rDNA gene sequencing. Group I comprised 26 isolates and was the second most abundant population, representing $33.3 \%$ of lactobacilli isolates; on MRS agar, isolates of this group formed white and bulging colonies of irregular shape. Sequenced strains of this group were identified as members of Lb. parakefiri species. Group II comprised 12 isolates that were identified as members of Lb. kefiri species. Growth on MRS agar revealed these isolates as white, round-shaped, and slightly bulging colonies. Group III contained 40 isolates and was the most abundant population, accounting for $51.3 \%$ of the lactobacilli isolates. These strains were identified as members of $L b$. kefiranofaciens ssp. kefirgranum that formed white, dry, compact, and bulging colonies of irregular shape on MRS agar.

Similar lactobacilli composition in kefir grains has been previously reported (Takizawa et al., 1998). Conversely, some authors indicated different microbial composition of kefir grains is probably due to factors such as the origin of kefir grains, the production method, the milk type used, and identification method. Moreover, some authors suggested that heterofermentative lactobacilli predominated over the homofermentative ones (Angulo et al., 1993), whereas others (Kandler and Kunath, 1983; Takizawa et al., 1998) indicated that only about $10 \%$ of lactobacilli in kefir grains were heterofermentative and $90 \%$ were homofermentative. The ratio between homofermentative lactobacilli, $L b$. kefiranofaciens ssp. kefirgranum, and heterofermenta- tive lactobacilli, Lb. kefiri and Lb. parakefiri, isolated from our kefir grains was almost 1:1. The main function of homofermentative lactobacilli in kefir grains is the production of lactic acid by fermentation of lactose, whereas heterofermentative lactobacilli are important for $\mathrm{CO}_{2}$ and ethanol production. Carbon dioxide contributes to the antimicrobial activity of lactic acid bacteria by replacing the existing molecular oxygen, creating an anaerobic environment; $\mathrm{CO}_{2}$ is also important for unique organoleptic properties of kefir. The gas produced leads to fine take coagulum formation and also imparts a sparkling mouth-feel to kefir (Koroleva, 1988). Takizawa et al. (1998) suggested that homofermentative lactobacilli, $L b$. kefiranofaciens ssp. kefiranofaciens, is able to produce large amounts of kefiran and is responsible for grain formation. However, in our study, Lb. kefiranofaciens ssp. kefiranofaciens was not detected by the methods used. Nevertheless, it is impossible to conclude with absolute certainty that this bacteria was not present in our kefir grains. Although some researchers found that Lb. kefiranofaciens ssp. kefiranofaciens on MRS agar under anaerobic conditions formed transparent, glossy, convex, tiny, and extremely slimy colony (Vancanneyt et al., 2004), we did not find colonies of that kind on MRS agar.

Findings obtained in one of our latest studies ( $\mathrm{T}$. Vardjan, I. Rogelj, and Čanžek Majhenič , unpublished data), suggest that kefiran is present in kefir grains; but none of the isolated lactobacilli strains from kefir grains was able to produce kefiran in pure culture, they only produced kefiran-like polysaccharides. Two possible reasons exist for this phenomenon, explaining why kefiran was present in kefir grains but no kefiran producers were detected. The first was that Lb. kefiranofaciens ssp. kefiranofaciens probably was present in our kefir grains, but we were unable to isolate it, most likely due to its low cell concentration. The second explanation might be that other lactobacilli were also capable of kefiran production, but kefiran production in pure culture was limited. Koroleva (1988) stated that kefir bacteria and yeasts, when separated from kefir grains and manipulated as pure cultures, either do not grow in milk or have a decreased biochemical activity, which further complicates the study of microbial populations of kefir grains.

The enumeration values obtained on KDM agar at first, second, third, and fourth sampling was 7.23, $7.33,7.21$, and $7.28 \mathrm{log} \mathrm{cfu} / \mathrm{g}$, respectively. Using KDM agar, a total of 42 yeasts were isolated and subjected to PCR-RAPD fingerprinting. Yeasts species were confirmed by a partial sequencing of the $26 \mathrm{~S}$ rDNA fragments. The culture-dependent approach indicated that Kl. marxianus represented the most abundant and commonly identified yeast (64\%), followed by $\mathrm{Ka}$. 
exigua (29\%) and Rhodosporidium kratochvilovae (7\%). On KDM agar, Kl. marxianus formed creamy, large, irregular, and umbonate colonies, whereas $\mathrm{Ka}$. exigua formed small, creamy, circular, and raised colonies. Rhodosporidium kratochvilovae formed large, circular, raised, and orange colonies. It is worth noting that the yeast species $R$. kratochvilovae, whose presence in kefir grains has not been reported previously, was detected in our study. The microbial community of kefir grains depends on their source and manipulation processes. The presence of $R$. kratochvilovae in our kefir grains may be due to contamination during manipulation process, or this strain is typical locally. To determine the exact origin of $R$. kratochvilovae in our kefir grains, further research must be completed. Kluyveromyces marxianus and $K a$. exigua isolated from our kefir grains have been previously isolated from kefir grains by other researchers (Garrote et al., 2001; Wang et al., 2008; Zhou et al., 2009). Farnworth and Mainville (2003) concluded that the list of prevalent yeasts in kefir grains from different parts of the world would not be very extensive, because a contaminant species would probably not survive due to the production of antimicrobial compounds by the symbiotic microbiota of kefir. When Witthuhn et al. (2004) used the Rapid ID 32C (API System S.A., Montalieu Vercieu, France) method to identify yeasts in South African kefir grains, Zygosaccharomyces spp. was the only genus isolated from traditional kefir grains and only mass cultivation of grains led to the growth of some other yeast, such as Candida lambica and Candida krusei. Nevertheless, yeast genera identified from different samples of kefir grains in their study differed from the yeast genera identified from kefir grains in our study. These differences clearly show that microbiota in kefir grains cannot be defined without taking into account factors such as the method of kefir production, the origin of kefir grains, and methods of microbial identification. Kluyveromyces marxianus is a lactosepositive yeast, whereas Ka. exigua and R. kratochvilovae are lactose-negative yeasts. Additionally $\mathrm{Kl}$. marxianus is able to use both lactose and galactose as its carbon source (Wang et al., 2008), which enables this yeast to multiply well in milk and probably explains why it is the primary yeast in kefir grains in our study and some other studies (Garrote et al., 2001; Wang et al., 2008; Zhou et al., 2009). Participation of Kl. marxianus in kefir grains ensures the metabolism of lactose through alcohol fermentation, and the formation of a typical yeasty flavor. Along with $\mathrm{Kl}$. marxianus, lactose negative yeasts also play very important roles in the formation of kefir flavor (Wang et al., 2008). Simova et al. (2002) reported that typical yeasty flavor and aroma were absent in kefir produced by Kl. marxianus only.

\section{Characterization of Lactobacilli and Yeasts in Kefir Grains Using Culture-Independent Method}

Conventional culture-dependent methods rely on plating procedures which are time-consuming, laborious, and provide limited information about microbial diversity in food samples. Namely, species occurring in low numbers are often out-competed in vitro by numerically more abundant species, and some species may not be able to grow in conditions provided (temperature, in vitro, $\mathrm{pH}$, medium composition, and so on). Lately, culture-independent methods have proven to be powerful tools in generating more complete data about microbial diversity in food matrices where PCR-DGGE is surely one of the most promising molecular techniques.

The isolated DNA from kefir grains (G1-G4), together with DNA of reference lactobacilli and yeasts (Lb. kefiranofaciens ssp. kefirgranum DSM $10550^{\mathrm{T}}, L b$. kefiranofaciens ssp. kefiranofaciens LMG $19149^{\mathrm{T}}$, Lb. kefiri LMG $9480^{\mathrm{T}}$ and S. cerevisiae CBS 1171, Ka. unispora NRRL Y-1556, Ka. exigua CBS 379, T. delbrueckii CBS 1146, and Kl. marxianus IM444), as well as pure cultures of lactobacilli, preliminarily isolated from kefir grains using the culture-dependent method (one strain from each group as follows: Lb. parakefiri KP91 from group I, Lb. kefiri MR3 from group II, and Lb. kefiranofaciens ssp. kefirgranum MP6 from group III) were used for PCR-DGGE analysis.

The V2-V3 region of the $16 \mathrm{~S}$ rRNA gene of bacteria was amplified and PCR products were analyzed by DGGE (Figure 1). In each serial sampling of kefir grains (G1-G4), one dominant band (band a) was more intensive and corresponded to the position of $L b$. kefiranofaciens ssp. kefirgranum DSM $10550^{\mathrm{T}}$ and $L b$. kefiranofaciens ssp. kefirgranum MP6, indicating that $L b$. kefiranofaciens ssp. kefirgranum was present in kefir grains. On the contrary, Lb. kefiri and Lb. parakefiri, whose presence were detected in all kefir grains by the culture-dependent method, were not detected with the PCR-DGGE method [i.e., no visible bands in the same position as band b (corresponding to Lb. kefiri LMG 9480 or Lb. kefiri MR3) and band c (corresponding to Lb. parakefiri KP91)]. Likewise, Da Cruz Pedrozo Miguel et al. (2010) detected Lb. helveticus and $L b$. parakefiri from kefir grains using the culture-dependent method, but not with the culture-independent method, which they explained by the fact that it could be difficult to obtain high-quality DNA suitable for PCR directly from the kefir grain samples. Another possible explanation might be that cell counts of certain lactobacilli species were lower than the detection limit of PCR-DGGE (Chen et al., 2008). Fasoli et al. (2003) define the sensitivity of PCR-DGGE for the detection 


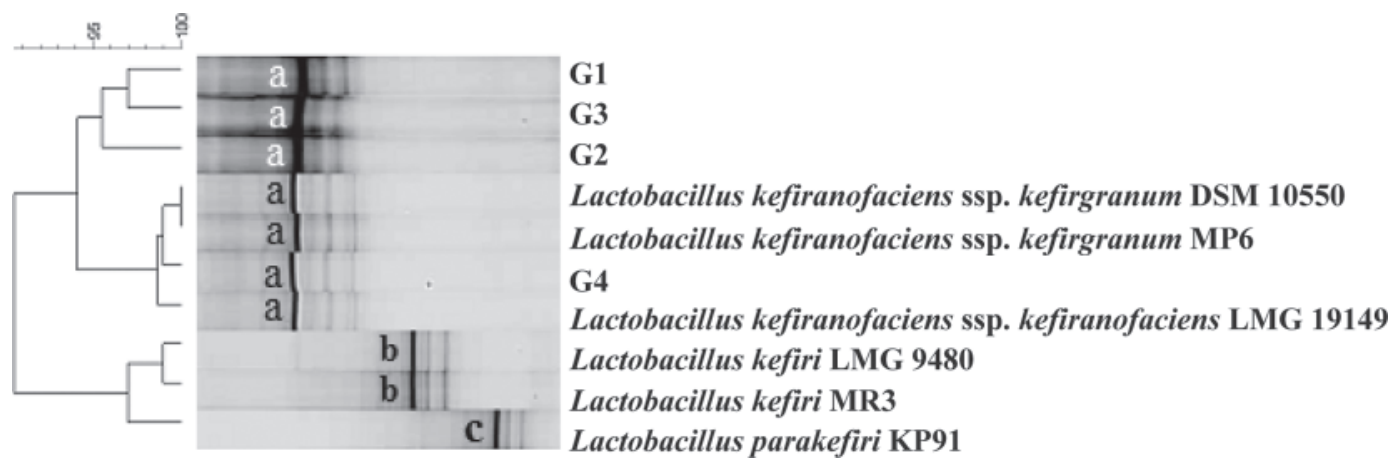

Figure 1. The denaturing gradient gel electrophoresis fingerprints of bacterial community present in kefir grains (G1-G4; samples of kefir grains), type strains (Lactobacillus kefiranofaciens ssp. kefirgranum DSM $10550^{\mathrm{T}}$, Lb. kefiranofaciens ssp. kefiranofaciens LMG $19149^{\mathrm{T}}$, and Lactobacillus kefiri LMG 9480 ${ }^{\mathrm{T}}$ ), and lactobacilli isolated from kefir grains using the culture-dependent method (Lb. kefiranofaciens ssp. kefirgranum MP6, Lb. kefiri MR3, and Lactobacillus parakefiri KP91). Bands: a $=$ Lb. kefiranofaciens ssp. kefirgranum; $\mathrm{b}=$ Lb. kefiri; and $\mathrm{c}=$ Lb. parakefiri. Color version available in the online PDF.

of the V2-V3 region in a complex environment with counts of bacterial population as high as $10^{7}$ to $10^{8}$ $\mathrm{cfu} / \mathrm{g}$. The limitation for the detection potential is a consequence of high quantities of competitor templates during the amplification reaction. Chen et al. (2008) investigated lactic acid bacteria in 3 different original kefir grains. They concluded that a combined approach, including cultivation, PCR-DGGE, and subsequent DNA sequencing, could successfully identify lactobacilli from kefir grains. Similar observations were also reported by Kesmen and Kacmaz (2011), as well as by the present study.

Figure 2 shows the PCR-DGGE fingerprints of the yeast community in kefir grains. All kefir grains (G1G4) samples had 2 common bands. Band d was in the same position as the band corresponding to $\mathrm{Ka}$. exigua CBS 379, and band c was in the same position as the band corresponding to Kl. marxianus IM444. These results indicated that $K a$. exigua and $K l$. marxianus were present in kefir grains. Species R. kratochvilovae, the least common yeast isolated from kefir grains, using the culture-dependent method, could not be identified by the culture-independent method using PCR-DGGE. This finding suggests that the species with a larger population in the mixture might give greater amounts of template DNA, and therefore make the detection of minor species difficult. Wang et al. (2008) identified 3 yeasts species, $K l$. marxianus, $S$. turicensis, and $P$. fermentans, from kefir grains using a combination of conventional microbiological cultivation method with PCR-DGGE. But they identified only 2 yeasts species, Kl. marxianus and S. turicensis, using the culture-independent method (PCR-DGGE). They concluded that, in samples containing multiple species, PCR-DGGE may fail to detect some species.

The PCR-DGGE analysis has been recognized as one of the most suitable and widely applied techniques for studying complex microbial communities originating from food samples, such as kefir grains (Zhou et al., 2009). However, it is worth stating that some limitations associated with the use of PCR-DGGE analysis of the microbial community structure exist. In our study, only $L b$. kefiranofaciens ssp. kefirgranum was detected in kefir grains using the culture-independent method (PCR-DGGE), whereas Lb. kefiri and Lb. parakefiri were detected only with the culture-dependent method. Yeast species $R$. kratochvilovae was also detected only with the culture-dependent method.

Furthermore, some lactobacilli were not distinguished using PCR-DGGE. Lactobacillus kefiranofaciens ssp. kefirgranum and Lb. kefiranofaciens ssp. kefiranofaciens had bands at the same position. The $16 \mathrm{~S}$ rDNA fragments with different sequences may have a similar melting behavior and co-migrate, and resulting bands at the same position in the gel are not necessarily

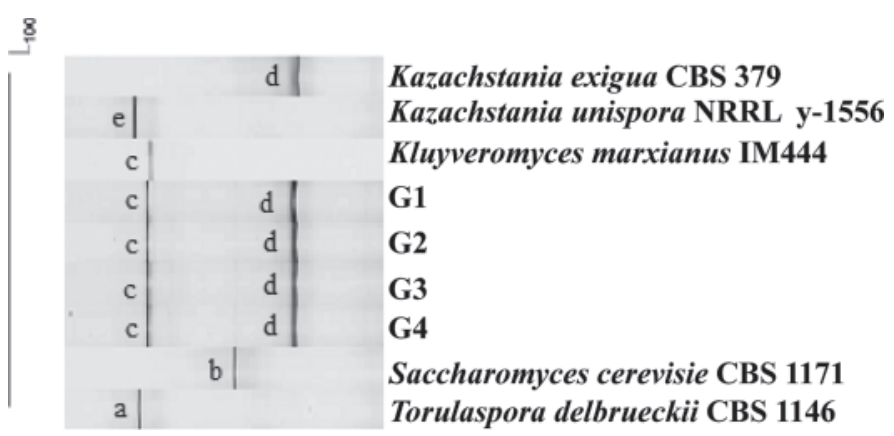

Figure 2. The denaturing gradient gel electrophoresis fingerprints of yeast community present in kefir grains (G1-G4; samples of kefir grains) and reference strains (Kazachstania exigua CBS 379, Kazachstania unispora NRRL y-1556, Kluyveromyces marxianus IM444, Saccharomyces cerevisiae CBS 1171, and Torulaspora delbrueckii CBS 1156). Bands: $\mathrm{a}=$ Torulaspora delbrueckii $; \mathrm{b}=$ Saccharomyces cerevisiae $; \mathrm{c}=$ Kluyveromyces marxianus $; \mathrm{d}=$ Kazachstania unispora; and $\mathrm{e}=$ Kazachstania exigua. Color version available in the online PDF 
phylogenetically related (Muyzer et al., 1993). Another problem was the formation of multiple band patterns for one species (Figure 1) instead of a single band that is representative of that particular species. The reasons for multiple banding within the one species are not well understood (Beh et al., 2006). Because of these problems, it would be reasonable to combine PCR-DGGE with sequencing. This would allow more accurate identification of species, but would extend the time and costs of analysis, by which PCR-DGGE analysis would lose most of its benefits.

\section{Microbial Stability of Kefir Grains}

Farnworth (2005) suggests that the industrial scale production of kefir with kefir grains can be limited due to variations in the composition of the microbiota of kefir grains, thus the production of consistent product is hindered. Therefore, kefir grains are usually replaced by starters composed of pure microorganisms that are isolated from kefir grains; but the quality of the product is quite different to that of kefir fermented with kefir grains (Farnworth and Mainville, 2003). Despite these claims, we assume that with proper manipulation of kefir grains its microbiota should retain a certain level of stability. Therefore, we decided to assess the microbial stability of kefir grains by using culture-dependent and culture-independent (PCR-DGGE) methods. In both approaches it has been demonstrated that size and composition of detected dominant lactobacilli and yeast in kefir grains were stable and did not change over experimental time. We found that the number of lactobacilli and yeasts from kefir grains was remarkably stable and did not change over serial sampling. Lactobacillus kefiranofaciens ssp. kefirgranum was the most abundant strain in all 4 serial samples of kefir grains using culture-dependent analysis, followed by $L b$. parakefiri. Furthermore, the PCR-DGGE profile of the bacterial and yeasts community present in kefir grains (Figure 1, Figure 2) also revealed that the prevailing microbiota of kefir grains did not change over serial sampling. This is the first report demonstrating that the typical microbial population of kefir grains retains stability during the manipulation of kefir grains on an industrial level, if it is done in accordance with good manufacturing and hygiene practices. We find this result important, because only a stable microbial community of kefir grains can contribute to high-quality and consistent product, which is also the case of Mlekarna Krepko dairy plant that produces kefir of high- and consistent quality by the use of same kefir grains that have been propagated since dairy plant establishment. But, to support our statement, further analysis with more frequent sampling over longer period of time are required.

\section{CONCLUSIONS}

The use of a combination of conventional microbiological cultivation techniques and molecular techniques (i.e., DNA sequencing) revealed 3 lactobacilli and 3 yeasts species in kefir grains; $L b$. kefiranofaciens ssp. kefirgranum, $L b$. parakefiri, and $L b$. kefiri were found in kefir grains with 51.3, 33.3, and $15.4 \%$ distribution, respectively. The most frequently isolated yeasts were $K l$. marxianus, Ka. exigua, and $R$. kratochvilovae, with 64, 29 , and $7 \%$ distribution, respectively. Furthermore, the PCR-DGGE culture-independent method was applied to identify lactobacilli and yeasts in kefir grains. Using this approach, Lb. kefiri, Lb. parakefiri, and R. kratochvilovae were not identified. This result confirms that only predominant lactobacilli and yeast populations in kefir grains could be identified. Therefore, the combination of both techniques is necessary for the detailed and reliable study of microbial communities in kefir grains. Descriptions of the different types of bacteria and yeast present in different batches of kefir grains have been provided by different authors; however, none of them analyzed the microbial stability of kefir grains. Using the culture-dependent and culture-independent methods, we have recognized that the detected dominant lactobacilli and yeasts of kefir grains were very stable over experimental time. This finding is important to ensure consistent product quality.

\section{ACKNOWLEDGMENTS}

This research was financed in part by the European Union Social Fund (Brussels, Belgium).

\section{REFERENCES}

Andrighetto, C., E. Psomas, N. Tzanetakis, G. Suzzi, and A. Lombardi. 2000. Randomly amplified polymorphic DNA (RAPD) PCR for the identification of yeasts isolated from dairy products. Lett. Appl. Microbiol. 30:5-9.

Angulo, L., E. Lopez, and C. Lema. 1993. Microflora present in kefir grains of the Galician region (North-west of Spain). J. Dairy Res. 60:263-267.

Beh, A. L., G. H. Fleet, C. Prakitchaiwattana, and G. M. Heard. 2006. Evaluation of molecular methods for the analysis of yeasts in food and beverages. Adv. Exp. Med. Biol. 571:69-106.

Beshkova, D. M., E. D. Simova, Z. I. Simov, G. I. Frengova, and Z. N. Spasov. 2002. Pure culture for making kefir. Food Microbiol. 19:537-544.

Chen, H. C., S. Y. Wang, and M. J. Chen. 2008. Microbiological study of lactic acid bacteria in kefir grains by culture-dependent and culture-independent methods. Food Microbiol. 25:492-501.

Cocolin, L., D. Aggio, M. Manzano, C. Cantoni, and G. Comi. 2002. An application of PCR-DGGE analysis to profile the yeasts populations in raw milk. Int. Dairy J. 12:407-411. 
Da Cruz Pedrozo Miguel, M. G., P. Gomes Cardoso, L. de Assis Lago, and R. F. Schwan. 2010. Diversity of bacteria present in milk kefir grains using culture-dependent and culture-independent methods. Food Res. Int. 43:1523-1528.

Dahllöf, I., H. Baillie, and S. Kjelleberg. 2000. rpoB-base microbial community analysis avoids limitations inherent in 16S rRNA gene intraspecific heterogeneity. Appl. Environ. Microbiol. 66:33763380 .

de Moreno de LeBlanc, A., C. Matar, E. Farnworth, and G. Perdigon. 2007. Study of immune cells involved in the antitumor effect of kefir in a murine breast cancer model. J. Dairy Sci. 90:1920-1928.

Farnworth, E. R. 2005. Kefir-A complex probiotic. Food Sci. Technol. Bull. Funct. Foods 2:1-17.

Farnworth, E. R., and I. Mainville. 2003. Kefir: A fermented milk product. Pages 77-103 in Handbook of Fermented Functional Foods. R. Farnworth, ed. CRC Press, Boca Raton, FL.

Fasoli, S., M. Marzotto, M. Rizzotti, L. Rossi, F. Dellaglio, and S. Torriani. 2003. Bacterial composition of commercial probiotic products as evaluated by PCR-DGGE analysis. Int. J. Food Microbiol. 82:59-70.

Garrote, G. L., A. G. Abraham, and G. L. De Antoni. 2001. Chemical and microbiological characterisation of kefir grains. J. Dairy Res. 68:639-652.

Garrote, G. L., A. G. Abraham, and G. L. De Antoni. 2010. Microbial interactions in kefir: A natural probiotic drink. Pages 327-336 in Biotechnology of Lactic Acid Bacteria: Novel Applications. F. Mozzi, R. R. Raya, G. M. Vignolo, ed. Wiley-Blackwell, Oxford, UK.

Hugenholtz, P., B. M. Goebbel, and N. R. Pace. 1998. Impact of culture independent studies on the emerging phylogenetic view of bacterial diversity. J. Bacteriol. 180:4765-4774.

Kandler, O., and P. Kunath. 1983. Lactobacillus kefir sp. nov., a component of the microflora of kefir. Syst. Appl. Microbiol. 4:286-294.

Kesmen, Z., and N. Kacmaz. 2011. Determination of lactic microflora of kefir grains and kefir beverage by using culture-dependent and culture-independent methods. J. Food Sci. 76:M276-283.

Klijn, N., A. H. Weerkamp, and W. M. De Vos. 1991. Identification of mesophilic lactic acid bacteria by using polymerase chain reactionamplified variable regions of $16 \mathrm{~s}$ rRNA and specific DNA probes. Appl. Environ. Microbiol. 57:3390-3393.

Koroleva, N. S. 1988. Technology of kefir and kumis. Bull. Int. Dairy Fed. 227:96-100.

Liu, J. R., M. J. Chen, and C. W. Lin. 2005. Antimutagenic and antioxidant properties of milk-kefir and soymilk kefir. J. Agric. Food Chem. 53:2467-2474.

Mainville, I., N. Robert, B. Lee, and E. R. Farnworth. 2006. Polyphasic characterization of the lactic acid bacteria in kefir. Syst. Appl. Microbiol. 29:59-68.

Marshall, V. M. 1993. Fermented milks. Pages 1804-1807 in Encyclopedia of Food Science, Food Technology and Nutrition. R. Macreae, R. K. Robinson, and M. J. Sadler, ed. Academic Press, London, UK.

Muyzer, G., E. C. de Waal, and A. G. Uitterlinden. 1993. Profiling of complex microbial populations by denaturing gradient gel electrophoresis analysis of polymerase chain reaction-amplified genes coding for 16S rRNA. Appl. Environ. Microbiol. 59:695-700.
Otes, S., and O. Cagindi. 2003. Kefir: A probiotic dairy-composition, nutritional and therapeutic aspects. Pakistan J. Nutr. 2:54-59.

Özer, D., and B. H. Özer. 1999. Product of Eastern Europe and Asia. Pages 798-805 in Encyclopedia of Food Microbiology. R. K. Robinson, ed. Academic Press, San Diego, CA.

Robinson, K. R., A. Y. Tamime, and M. Wszolek. 2002. Microbiology of fermented milks. In Dairy Microbiology Handbook. B. J. B. Wood, ed. Wiley-Interscience, New York, NY.

Rodrigues, K. L., L. R. Gaudino Caputo, J. C. Tavares Carvalho, J. Evangelista, and J. M. Schneedorf. 2005. Antimicrobial and healing activity of kefir and kefiran extract. Int. J. Antimicrob. Agents 25:404-408.

Santos, A., M. San Mauro, A. Sanchez, J. M. Torres, and D. Marquina. 2003. The antimicrobial properties of different strains of Lactobacillus spp. isolated from kefir. Syst. Appl. Microbiol. 26:434-437.

Simova, E., D. Beshkova, A. Angelov, T. Hristozova, G. Frengova, and Z. Spasov. 2002. Lactic acid bacteria and yeasts in kefir grains and kefir made from them. J. Ind. Microbiol. Biotechnol. 28:1-6.

Takizawa, S., S. Kojima, S. Tamura, S. Fujinaga, Y. Benno, and T. Nakase. 1998. The composition of the lactobacillus flora in kefir grains. Syst. Appl. Microbiol. 21:121-127.

Toba, T., S. Abe, K. Arihara, and S. Adachi. 1986. A medium for the isolation of capsular bacteria from kefir grains. Agric. Biol. Chem. 50:2673-2674.

Torriani, S., G. Zapparoli, and F. Dellaglio. 1999. Use of PCR-based methods for rapid differentiation of Lactobacillus delbrueckii ssp. bulgaricus and L. delbrueckii ssp. lactis. Appl. Environ. Microbiol. 65:4351-4356

Valderrama, M. J., M. I. De Siloniz, P. Gonzalo, and J. M. Peinado. 1999. A Differential medium for the isolation of Kluyveromyces marxianus and Kluyveromyces lactis from dairy products. J. Food Prot. 62:189-193.

Vancanneyt, M., J. Mengaud, I. Cleenwerck, K. Vanhonacker, B. Hoste, P. Dawyndt, M. C. Degivry, D. Ringuet, D. Janssens, and J. Swings. 2004. Reclassification of Lactobacillus kefirgranum Takizawa et al. 1944 as Lactobacillus kefiranofaciens ssp. kefirgranum ssp. nov. and emended description of L. kefiranofaciens Fujisawa et al. 1988. Int. J. Syst. Evol. Microbiol. 54:551-556.

Viljoen, B. C. 2001. The interaction between yeasts and bacteria in dairy environments. Int. J. Food Microbiol. 69:37-44.

Walter, J., G. W. Tannock, A. Tilsala-Timisjarvi, S. Rodtong, D. M. Loach, K. Munro, and T. Alatossava. 2000. Detection and identification of gastrointestinal Lactobacillus species by using denaturing gradient gel electrophoresis and species-specific PCR primers. Appl. Environ. Microbiol. 66:297-303.

Wang, S. Y., H. C. Chen, J. R. Liu, Y. C. Lin, and M. J. Chen. 2008. Identification of yeasts and evaluation of their distribution in Taiwanese kefir and viili starters. J. Dairy Sci. 91:3798-3805.

Witthuhn, R. C., T. Schoeman, and T. J. Britz. 2004. Isolation and characterization of the microbial population of different South African kefir grains. Int. J. Dairy Technol. 57:33-37.

Zhou, J., X. Liu, H. Jiang, and M. Dong. 2009. Analysis of the microflora in Tibetan kefir grains using denaturating gradient gel electrophoresis. Food Microbiol. 26:770-775. 